ISSN: $1130-3743$ - e-ISSN: 2386-5660

DOI: http://dx.doi.org/10.14201/teoredu2016282225248

\title{
APRENDER Y SERVIR EN LA UNIVERSIDAD: UNA VÍA CÍVICA AL DESARROLLO EDUCATIVO
}

\section{To learn and to serve in the university: a civic pathway to the educational development}

\section{Apprendre et servir à l'université: une voie vers le développement de l'éducation}

\author{
Alexandre Sotelino Losada, Miguel Ángel Santos Rego \\ y María del Mar LORENZO MOLEDO \\ Facultad de Ciencias de la Educación. Departamento de Teoría de la Educación, \\ Historia de la Educación y Pedagogía Social. C/ Xosé M. a Suárez Núñez. \\ Campus Vida, 15782. Santiago de Compostela (A Coruña). \\ alexandre.sotelino@usc.es; miguelangel.santos@usc.es; mdelmar.lorenzo@usc.es
}

Fecha de recepción: enero de 2016

Fecha de aceptación: marzo de 2016

\section{RESUMEN}

En este artículo se examina si algunos cambios asociados al Espacio Europeo de la Educación Superior (EEes) han afectado a los procesos educativos en la universidad, toda vez que la reforma implica un enfoque del aprendizaje centrado en la adquisición de competencias por parte de los estudiantes. Su objetivo no es otro que explorar las posibilidades que para la formación del alumnado tiene la conexión entre universidad y comunidad. A tal fin, después de revisar la literatura temática de los últimos años, hacemos uso de un trabajo empírico con metodología ex-postfacto. Y se propone el aprendizaje-servicio como estrategia capaz de favorecer una pedagogía más experiencial en la universidad. 
Palabras clave: educación; universidad; competencias; compromiso cívico; aprendizaje-servicio.

\section{SUMMARY}

This paper examines whether some changes associated with the European Higher Education Area (EHEA) have affected the educational processes at the university, while also the reform involves a learning approach focused on the acquisition of skills by students. Its objective is to explore the possibilities for the training of students is the connection between university and community. To that end, after review of the thematic literature of recent years, we use an empirical study with ex-post-facto methodology. And we propose service-learning as a strategy able to favour a more experiential pedagogy at the university.

Key words: education; university; competencies; civic engagement; service-learning.

\section{SOMMAIRE}

Cet article analyse si certains des changements liés à l'Espace Européen de l'Éducation Supérieur (EEES) ont eu des effets sur les processus éducatifs à l'Université, alors que la réforme implique une approche d'apprentissage concentrée sur l'acquisition des connaissances de la part des étudiants. L'objectif est d'étudier les possibilités que le lien entre l'Université et la communauté offre pour la formation des étudiants. Pour cela, avoir révisé la littérature thématique des dernières années, nous utilisons un travail empirique avec méthodologie ex-post-facto. On propose l'apprentissage-service comme une stratégie capable de favoriser une pédagogie plus expérientielle à l'Université.

Mots clés: éducation; université; compétences; engagement civique; apprentissage-service.

\section{INTRODUCCIÓN}

Desde el mismo inicio de siglo, ante la implantación del Espacio Europeo de Educación Superior (EEES), muchas universidades españolas han iniciado programas de formación continua de su personal docente, que en algunos casos se han acompañado de convocatorias de proyectos para la mejora de la enseñanza y, sobre todo, el aprendizaje de sus estudiantes.

Se puede afirmar que en este proceso no todo ha sido búsqueda de diplomas acreditativos en respuesta a dinámicas institucionales de aseguramiento de la calidad (Santos Rego, 2010). También son muchas las profesoras y profesores que han demostrado su capacidad de generar dinámicas de aprendizaje más efectivas para 
ampliar las posibilidades de éxito de los estudiantes, haciéndolo más interactivo, vinculándolo a casos reales, aprovechando la tecnología digital, o basándolo en la resolución de problemas.

En este sentido, se debe recordar que la Declaración de Bolonia de 19 de junio de 1999 fue realizada bajo principios de calidad, movilidad, diversidad y competitividad, y orientando sus propósitos hacia la consecución, entre otros, de dos objetivos estratégicos: el incremento del empleo en la Unión Europea y la conversión del sistema Europeo de Formación Superior en un polo de atracción para estudiantes y profesores de otras partes del mundo (Zabalza, 2011). Este nuevo marco europeo prestaba también atención a la posibilidad de una nueva ciudadanía europea a través de la educación, pero sin desprecio hacia las particularidades de los diferentes Estados. Así, se ponía un cierto acento en el desarrollo de habilidades sociales y relacionales, junto a la promoción de una cultura educativa basada en el principio de "aprender a aprender» que tanto citó Delors (1994) en su célebre informe para la Comisión Europea. Los egresados de las Universidades europeas tendrán que conocer los principales contenidos de su titulación, pero también tendrán que estar formados en valores, capacidades y habilidades para funcionar adecuadamente en la comunidad. Aun con una perspectiva distante seguimos debatiendo el modelo de universidad europea, algo que quizás se derive del pesimismo apoyado en la creencia de que no es posible el cambio apuntado dada la escasez de medios y la dificultad de su adaptación, dejando algunas titulaciones al margen del proceso, como es el caso de Derecho en Alemania (Lorenzo, 2012). La comunidad universitaria, en palabras del profesor Sanjurjo $(2012,123)$, ha "asumido entre la resignación descreída y el entusiasmo entregado las tesis de Bolonia».

Lo que parece cierto es que el EEEs ha reforzado la representación colectiva de una universidad que, además de cualificar profesionalmente, también se debe comprometer con la formación de profesionales competentes, social y cívicamente hablando (Gaete, 2011). Así, uno de los retos más urgentes del EeEs es la formación de una ciudadanía dispuesta a participar activamente en el fortalecimiento de la sociedad civil y en el desarrollo de una ciudadanía participativa y comprometida con los problemas del presente y los retos del futuro (Santos Rego y Lorenzo, 2010; Santos Rego, 2016).

Siguiendo al profesor Martínez Martín (2006), la universidad tiene una función ética que se proyecta en tres dimensiones: la formación deontológica; la formación ciudadana y cívica de los estudiantes, y la formación humana, personal y social a favor de una capacitación ética y moral de los titulados. Para ello, desde la educación superior se deben trazar nuevas vías que ayuden a hilvanar estas tres dimensiones y redundando en última instancia en la calidad educativa, puesto que no se debe olvidar que la universidad es, ante todo, una institución formadora. Justamente, en los últimos años parece ir en aumento una cierta sensibilidad respecto de la necesidad de promocionar los aspectos cívico-sociales en el ámbito universitario. Esa preocupación se explica tanto desde parámetros político-institucionales, como desde exigencias del mismo mercado laboral (Jover y González Geraldo, 2013). 
ALEXANDRE SOTELINO, MIGUEL ÁNGEL SANTOS Y MARÍA DEL MAR LORENZO APRENDER Y SERVIR EN LA UNIVERSIDAD: UNA VÍA CÍVICA AL DESARROLLO EDUCATIVO

Se da por hecho que en la universidad se sabe diseñar y realizar magníficos proyectos de investigación. Pero se silencia más de lo debido que esa pericia va unida a una aptitud para integrar aprendizaje y necesidades sociales, que es donde se sitúa la clave del éxito (cfr. Lucas, 2009). El corolario es, pues, automático: cuanto más conocimiento y destreza transfieran los estudiantes desde el aula a la comunidad, y viceversa, mejor preparados estarán para afrontar lo que les ha de venir en su vida personal y profesional.

En este trabajo nos fijamos como objetivo contribuir al examen de las vías que vienen articulando la relación entre universidad y sociedad o, si se prefiere, entre universidad y comunidad, a fin de proponer, con criterio de mayor efectividad, otras estrategias que permitan visualizar la nueva realidad universitaria. Este es el caso del aprendizaje-servicio.

\section{LA UNIVERSIDAD Y EL CAPITAL SOCIAL}

Este afán de la universidad en construir ciudadanos debería aportar buenos resultados porque produce capital social, y es bien sabido que la teoría del capital social se fundamenta en la creencia del valor que proporcionan las relaciones entre las personas, las cuales inciden en el cambio de los individuos hacia la acción. El papel de la educación superior no se ha de limitar únicamente a la mejora del crecimiento económico por los efectos sobre el capital humano, que también, sino que afectará a la autoestima y a la libertad de las personas (Cabrera, 2000). Se puede afirmar que no habrá cambio si no existe solidaridad, lo que implica sentido de pertenencia a una estructura social (Saiz y Jiménez, 2008).

Ese capital social del que hablamos se alimenta de una serie de componentes de corte cualitativo, como pueden ser las mutuas obligaciones, la reciprocidad y la misma cooperación en la procura de un beneficio común. Podría entenderse como una red de estructuras interrelacionadas que ayuda a mantener el estado de bienestar (Vernon y Foster, 2002). Como señalan García Roca y Mondaza (2002), la universidad posee un capital social que la sitúa en ventaja comparativa y le otorga una responsabilidad ante la sociedad, residiendo su propia fortaleza en los siguientes factores:

- Poseer personal técnicamente preparado, que quiere abrirse a la cooperación, en cada una de las especialidades científicas.

- Disponer de una apreciable conciencia solidaria entre los universitarios que buscan canalizar sus aptitudes de cara a la cooperación.

- Estar en condiciones de promover la integralidad de la intervención más allá de sus respectivos sectores representados por las distintas asociaciones de ingenieros, arquitectos, médicos, farmacéuticos o pedagogos sin fronteras.

En este sentido, el concepto de responsabilidad social universitaria tendrá que adquirir nuevos matices si queremos que cumpla con el objetivo de extender el conocimiento a la sociedad. Conviene más complicidad con las comunidades que 
conforman el tejido social próximo a la institución universitaria. Al fin y al cabo, lo que se pretende es ayudar a construir la nueva ciudadanía de un país que aspira a una democracia de mayor calidad y con más equidad cuando el aprendizaje de la participación se ha convertido en pieza básica de su armazón axiológico. Lo que podría rotularse como "virtudes cívicas», a enumerar en la vida universitaria, no deben confundirse con una especie de canon moral de obligada referencia, o que deba ser transmitido, pues eso, aparte de otras consideraciones, mermaría la posibilidad de reformular las mismas disposiciones delante de nuevas necesidades o en el marco de problemas muy concretos. Citando a Barber (1998), no es que la universidad tenga una misión cívica, sino que la universidad es una misión cívica, es civilidad en sí misma, matiza, que se define a través de las reglas y convenciones que facilitan el diálogo de una comunidad y la clase de discurso del que todo conocimiento depende.

La universidad es institución educativa y, como tal, debe vincularse a su entorno social inmediato, no debe ser una isla en el extenso océano social, sino que ha de constituir un elemento más del mismo y, por supuesto, uno de sus principales núcleos dinamizadores. Esta relación se hace incluso necesaria para desarrollar su función investigadora, porque las realidades de las que parte y a las que transfiere los resultados del conocimiento que genera son, habitualmente, las más inmediatas. En lo que a España se refiere, esto ya aparece recogido en el título preliminar de la Ley Orgánica 6/2001, del 21 de diciembre ( $B O E$ 24/12/2001), de Universidades, modificada por la Ley Orgánica 4/2007 del 12 de abril de 2007 (BOE 13/04/2007) donde se destacan las funciones de la universidad (art. 2):

a) La creación, desarrollo, transmisión y crítica de la ciencia, de la técnica y de la cultura.

b) La preparación para el ejercicio de actividades profesionales que exijan la aplicación de conocimientos y métodos científicos y para la creación artística.

c) La difusión, la valorización y la transferencia del conocimiento al servicio de la cultura, de la calidad de la vida y del desarrollo económico.

d) La difusión del conocimiento y la cultura a través de la extensión universitaria y la formación a lo largo de toda la vida.

Repárese en que el texto legal afirma "funciones al servicio de la sociedad», lo cual certifica que la universidad tiene un claro compromiso social. Por lo tanto, hoy más que nunca, la universidad ha de potenciar las posibilidades de cada alumno en un mundo abierto y cosmopolita, redefiniendo las capacidades y disposiciones a fortalecer en la medida que el proceso de aprendizaje puede beneficiarse de lo mucho que ya sabemos sobre cognición situada.

Pero de la universidad y de los aprendizajes que proporciona o alienta, todos esperamos más y mejores indicadores, que no solo tienen que ver con el desarrollo científico-tecnológico de un país, sino también con el aumento de la equidad social, de las oportunidades para las personas, independientemente de su género 
o adscripción étnico-cultural (Núñez Cubero, 2015). La universidad sigue siendo un depósito de esperanza para muchas familias, que la perciben como una vía de mejora para su vida y la de su descendencia. Y no es nada extraño ver cómo tales augurios son positivamente corroborados en los estudios, de índole sociológica, tanto en América como en Europa, sobre el grado de confianza que les merecen a los ciudadanos distintas instituciones (Santos Rego, 2013).

Es palmario que la relación sociedad-universidad ha de ser fluida y pragmática, en el sentido de aligerar los vínculos teóricos y poco efectivos. Así, la comunidad universitaria tiene que participar de la vida social, sumergirse en ella, y convertir en oportunidades sus necesidades y problemas. De la misma forma, la sociedad civil debe ver en la universidad una institución afín e indisociable de sus mejores propósitos (Santos Rego y Lorenzo, 2007). En definitiva, una agencia útil a la ciudadanía en una democracia fuerte (Santos Rego, 2013).

Ahora bien, la universidad tiene establecidas sus propias vías institucionales de relación con el medio social. Que no son iguales en las diferentes latitudes pues tampoco son idénticas las filosofías y las estructuras de gestión administrativas. En nuestras proximidades, los Consejos Sociales, como órganos de gobierno colegiados, sirven como teórico punto de encuentro entre la sociedad civil y la universidad, de tal manera que se facilita la comunicación y colaboración entre ambas instancias, para ayudar al desarrollo social, económico, cultural, educativo, laboral y tecnológico. Nacen como tal a partir de la Ley Orgánica de Universidades de 2001, que los define como el órgano de participación de la sociedad en la universidad.

Si tomamos como ejemplo el caso que nos es más cercano, sus funciones se han concretado en la Ley 1/2003, de 9 de mayo (DOG 30/05/2003), de los Consejos Sociales del Sistema Universitario de Galicia. Esa ha sido la ocasión para dilatar un poco su definición. Se le concibe como órgano de participación de la sociedad gallega en las universidades públicas que integran el sistema universitario de esta Comunidad. Su cometido ha de ser el de impulsar la colaboración entre Sociedad y Academia. Para ello, las universidades se esforzarán por satisfacer las necesidades de su entorno, contribuyendo eficazmente al desarrollo social, profesional, económico, tecnológico y cultural de Galicia; al avance de la calidad del servicio público de la educación superior universitaria, y a la obtención de los recursos precisos para procurar su suficiencia económica y financiera.

Llegados a este punto son varias las cuestiones que, legítimamente, preocupan. Entre ellas, las tres siguientes: ¿Es suficiente este vínculo entre ambas instancias? ¿Debemos buscar vías más pragmáticas que ayuden a (re)construir la sociedad civil? ¿Cómo se llevará esto a cabo y cómo se evaluará?

En cuanto a la suficiencia del vínculo, la respuesta no puede ser positiva, en tanto que los Consejos Sociales, aun suponiendo un pequeño aliciente, no conforman, aqui y ahora, una instancia con posibilidades suficientes (Larran, 2014). También los propios centros y departamentos universitarios están llamados a promover avances en este sentido. Y los mismos planes de estudios tienen que contemplar 
metodologías capaces de vincular los aprendizajes que sean menester con el contexto social y comunitario. Lo que precisamos son estudiantes universitarios preparados y dispuestos a participar activamente en la sociedad y a comprometerse en el análisis y progresiva resolución de sus problemas. Pero es cierto, como advierte Campo $(2008,89)$, que a menudo somos demasiado optimistas, pues esperamos que los ciudadanos participen y se comprometan de manera libre y espontánea, sin caer en la cuenta de que esos aprendizajes requieren de oportunidades educativas que los faciliten.

\section{RE-BUSCANDO LA CIUDADANÍA EN LA EDUCACIÓN SUPERIOR}

Si nos fijamos en las demandas, anteriormente expuestas, del Espacio Europeo de Educación Superior, veremos que lo que se reclama es que los estudiantes sean más activos en el ámbito académico y social, protagonizando su formación con carácter proactivo. Ello requiere un trabajo firme con asesoramiento y seguimiento permanente de los profesores, a los que corresponde ser orientadores del proceso educativo. Esta implicación de los estudiantes en su propio proceso de aprendizaje está recogida en la reforma de la Ley Orgánica 4/2007, de 12 de abril, por la que se modifica la Ley Orgánica 6/2001, de 21 de diciembre, de Universidades (LOMLOU) situando a los estudiantes como epicentro del sistema educativo y ofreciendo un marco institucional y reglado para su participación en la vida universitaria, contemplando el marco del Estatuto del Estudiante Universitario que ampara la creación del Consejo del Estudiante Universitario.

El Estatuto del Estudiante Universitario aprobado por Real Decreto 1791/2010, de 30 de diciembre, establece mecanismos y diferentes posibilidades para aumentar la implicación de los estudiantes en la vida universitaria y su participación y gestión en los servicios universitarios. Se otorga valor añadido a la implicación del estudiantado en la gestión de la universidad como institución pública, entendiendo esta como tarea de responsabilidad compartida; es lo que se afirma en el artículo que pasamos a transcribir:

Artículo 34. Principios generales. La universidad, como proyecto colectivo, debe promover la participación de todos los grupos que la integran. Los estudiantes, protagonistas de la actividad universitaria, deben asumir el compromiso de corresponsabilidad en la toma de decisiones, participando en los distintos Órganos de Gobierno a través de sus representantes democráticamente elegidos.

Otro de los pilares principales de este texto está en la valoración que se hace de la participación en actividades de tipo cultural, deportivo y/o solidario como parte de la vida universitaria. Entendiendo así la educación universitaria de manera holística, como el compendio de habilidades, conocimientos y actitudes que capacitan para el ejercicio profesional y ciudadano. Lo cual supone la superación de una concepción clásica de la universidad como institución cerrada y al margen de toda racionalidad que no sea la puramente científico-tecnológica. Ahora se 
propugna una Universidad menos determinista y más sensible a los problemas del entorno. Así lo recoge en el artículo 5: "Cualificaciones académicas y profesionales. [...] las universidades incorporarán a sus objetivos formativos la formación personal y en valores".

En el Capítulo XIII se especifica la importancia de la formación en valores, dado que la universidad debe ser un espacio de formación integral no solo para sus estudiantes sino para todas las personas que en ella conviven. La actividad universitaria debe promover las condiciones para que los estudiantes sean personas autónomas, aptos para tomar sus decisiones y actuar en consecuencia; responsables, dispuestos a asumir sus actos de modo responsable. Puesto que se trata de formar a los profesionales, la preocupación básica es la de facilitarles vías para el afrontamiento de la realidad. El Estatuto lo recoge de manera clara en su artículo 63.2 «De la formación en valores: Principios generales» donde se dice:

2. La actividad universitaria debe promover las condiciones para que los estudiantes: a) sean autónomos, aptos para tomar sus decisiones y actuar en consecuencia; b) sean responsables, dispuestos a asumir sus actos y sus consecuencias; c) sean razonables, capaces de procurar su propio bien y armonizar esta búsqueda con la de los otros; d) tengan sentido de la justicia, conocedores de la legalidad y prestos a dirimir racionalmente, con objetividad e imparcialidad, las diferencias con los otros implicados; e) tengan capacidad para incluir en su ámbito de responsabilidad a todos los otros afectados por sus elecciones y sus actuaciones, en especial la de aquellos que tienen menos capacidad para hacer valer sus intereses o mostrar su valor.

Tenemos que destacar también la creación del Consejo del Estudiante Universitario como otro tema de interés en el Estatuto del Estudiante Universitario. Este se recoge con amplitud en el Capítulo XI del texto. La puesta en marcha de este nuevo órgano es de gran importancia porque establecerá un canal directo de representación para todos los estudiantes, semejante al que tienen los rectores y las Comunidades Autónomas a través del Consejo de Universidades y de la Conferencia de Política Universitaria, y fortalecerá el papel central de los estudiantes dentro del sistema universitario español. Este órgano de representación tendrá una visibilidad institucional y abrirá una vía de comunicación paralela y complementaria de las asociaciones estudiantiles; por tanto, formará parte del marco clave para debatir las políticas de modernización y desarrollo de la convergencia europea. Entre las funciones de este órgano están las siguientes (ver www.mecd.gob.es):

- Representar al conjunto de los estudiantes de las Universidades Españolas y ser un interlocutor válido ante el Ministerio de Ciencia e Innovación.

- Contribuir activamente a la defensa de los derechos de los estudiantes, cooperando con [...] los órganos de representación estudiantil.

- Velar por la adecuada actuación de los órganos de gobierno de las Universidades en lo que se refiere a los derechos y deberes de los estudiantes.

- Recibir y, en su caso, dar cauce a las quejas que le presenten los estudiantes universitarios. 
- Colaborar con los Defensores Universitarios, en garantía de los derechos de los estudiantes de las Universidades españolas.

- Fomentar el asociacionismo estudiantil, y la participación de los estudiantes en la vida universitaria.

Atendiendo a un punto nuclear del artículo, el aprendizaje-servicio, este texto contempla por primera vez en un documento oficial del Estado la posibilidad de desarrollar una metodología de tal alcance en la universidad, como vía para fomentar la participación social y la cooperación al desarrollo de los estudiantes. Tanto es así, que en el Capítulo XIV «De las actividades de participación social y cooperación al desarrollo de los estudiantes", en el artículo 64.3 se dice lo siguiente:

Las universidades deberán favorecer la posibilidad de realizar el prácticum (obligatorio en algunas titulaciones y voluntario en otras) en proyectos de cooperación al desarrollo y participación social en los que puedan poner en juego las capacidades adquiridas durante sus estudios, lo que implica el derecho al reconocimiento de la formación adquirida en estos campos.

De igual forma favorecerán prácticas de responsabilidad social y ciudadana que combinen aprendizajes académicos en las diferentes titulaciones con prestación de servicio en la comunidad orientado a la mejora de la calidad de vida y la inclusión social.

Como vemos, es palpable la referencia a la posibilidad de realizar un servicio que tenga relación con los aprendizajes académicos. Creemos que este punto, junto a la afirmación de que precisamos estudiantes más autónomos, participativos y solidarios, supone todo un soporte normativo para la instauración e implementación del aprendizaje-servicio en nuestras universidades.

En definitiva, puestos a destacar un eje de atención del texto que comentamos, este sería el de la formación en valores para los alumnos universitarios. Tales valores, vinculados al respeto, la tolerancia, el reconocimiento de la diversidad, la libertad, la equidad y la solidaridad, han de expresarse de modo que sea posible asociarlos al proceso de participación social, pero también al aprendizaje académico, sin merma del esfuerzo y el rendimiento de cuentas individual. En palabras de Martínez-Odria (2010, 18), su actuación deberá estar presidida por «la honradez, la veracidad, el rigor, la justicia, la eficiencia, el respeto y la responsabilidad».

Hemos, pues, de intentar formar a los estudiantes universitarios según modos y maneras capaces de fortalecer una escala de valores sociales y de actitudes coherentes, considerando la formación autónoma de la personalidad y con especial atención a las experiencias sociales. Porque lo cabal será procurar una ciudadanía más consciente y más activa, más adaptable y competitiva en un entorno social y económico cambiante, al tiempo que más solidaria y crítica. Los valores de los que hablamos se aprenden, mayormente, en la cotidianeidad, en los marcos de convivencia entre personas, grupos e instituciones. La universidad no puede hacer caso 
omiso de este hecho, por más que sigan abundando quienes creen que la formación ciudadana y en valores democráticos no es responsabilidad de la universidad. Sin embargo, hay muchas prácticas unidas a la formación que se dispensa en las aulas, seminarios o laboratorios universitarios que contienen ingredientes morales de gran expresividad (Puig, 2003).

Vivimos tiempos convulsos y la incertidumbre se ha convertido en referencia común de nuestros discursos. Razón suficiente para ir aprendiendo a cooperar más y mejor dentro de nuestras respectivas unidades de pensamiento y acción, alentando el diseño de proyectos que impliquen a instancias de la sociedad civil. Es así como antaño se han apurado los cauces del cambio y del progreso y es así como podemos seguir explorando el potencial de la universidad para mejorar las condiciones de vida de la gente en las sociedades conectadas de hoy.

De todos modos, y siguiendo el hilo del trabajo, la universidad debe formar y preparar para el ejercicio activo de la democracia ya que una de sus fundamentales preocupaciones es la esfera de lo público, de lo colectivo, con uno de sus ejes girando alrededor del análisis y resolución de los problemas individuales y grupales. En otras palabras, en la universidad tiene que empezar a cobrar carta de naturaleza educativa la idea de una pedagogía para la inclusión y la responsabilidad civil (Santos Rego y Lorenzo, 2010). Por lo tanto, y ante los retos planteados, lo que debe orientar las políticas educativas universitarias es la formación de ciudadanas y ciudadanos:

- Activos, lo cual ha de servir para reforzar la participación como gran valor cívico (ciudadanía activa).

- Responsables, lo que permite defender con firmeza la responsabilidad social como valor cívico (ciudadanía responsable).

- Abiertos a la diversidad cultural, esto es, dispuestos a la racionalidad dialógica y a la puesta en valor de lo común en la vida cívica (ciudadanía intercultural).

- Comprometidos con la equidad de género en el espacio público como valor cívico (ciudadanía paritaria).

- Respetuosos con el medio ambiente y la promoción de la sostenibilidad como valor sustantivo (ciudadanía ambiental).

Las competencias nos hablan del conjunto de destrezas que todas las personas precisan para integrarse en todos los ámbitos de la sociedad. Para el desarrollo de las competencias debemos tener en cuenta un aspecto, como el de la contextualización de los aprendizajes. Recordemos que las competencias básicas son «[...] aquellas que todas las personas precisan para su realización y desarrollo personales, así como para la ciudadanía activa, la integración social y el empleo» (Comisión de las Comunidades Europeas, 2006). Para las profesoras Ugarte y Naval (2008-09) de la Universidad de Navarra, la competencia cívica supone, fundamentalmente, dos aspectos básicos, ser crítico y participativo. Y explican por qué: 
1. Crítico. De entre todas las competencias que facilitan la consolidación del pensamiento crítico en los estudiantes, las autoras consideran que uno de los medios más adecuados es la toma de decisiones, ya que se basa en la observación, el análisis y la reflexión. La decisión es el resultado de alcanzar una conclusión ante un problema presentado. Supone la elección racional entre alternativas, una vez valoradas. Analizados las ventajas y los inconvenientes de una decisión, son necesarios diligencia y coraje para actuar. Pero para la toma de decisiones acertadas y coherentes hay que ejercitar otras dos competencias: el liderazgo personal y la integridad.

2. Participativo. Para contribuir a la mejora social, no es suficiente con adquirir y consolidar un pensamiento reflexivo y crítico. Es preciso transmitir la necesidad de participar en las cuestiones sociales y cívicas como vía para buscar respuestas a los problemas y retos sociales. La toma de decisiones puede ser una competencia que no solo facilita la reflexión y el análisis, sino también la participación social.

Entre las competencias participativas, las citadas investigadoras destacan el trabajo en equipo, la capacidad de iniciativa y las habilidades de comunicación, de negociación o de resolución de problemas.

Lo que parecía una tendencia a la "titulitis" - permítasenos la licencia de tan informal vocablo-, entendiendo por tal una obsesión por la acumulación de acreditaciones y títulos de diferente índole, está siendo desplazada por el propio mercado laboral. Lo decimos porque ahora ya han ido adquiriendo fuerza otros factores como los desarrollados en las competencias anteriormente expuestas. Así, habilidades de carácter sociorrelacional, la iniciativa personal, la capacidad de reciclaje profesional son aspectos especialmente apreciados por los directivos de las empresas para la contratación de nuevos profesionales e incluso para la renovación de contratos.

Siguiendo esta línea discursiva, no nos debemos olvidar de que el sistema educativo debe preparar a nuestros jóvenes para los ámbitos social y laboral; aunque en la mayoría de las ocasiones es en el segundo de los factores donde se hace mayor hincapié, descuidando la función cívica de la institución. En pleno siglo XXI, parece que la universidad sigue reproduciendo valores añejos y no avanza a la par que lo hacen la sociedad y el mercado laboral. No estamos diciendo que la universidad se ponga al servicio del sistema de mercado, pero sí afirmamos la conveniencia de un mínimo de consistencia con los avances y cambios sociales vigentes.

Para ir cerrando este apartado, cabe resaltar que aprendizaje y participación social en la universidad no se pueden definir como un binomio en el proceso educativo ya que, tradicionalmente, el papel del alumno ha sido predominantemente pasivo, un simple receptor de conocimiento. En este sentido, se ha visto cómo la Academia ha ido tomando conciencia de la importancia que tiene la implicación social de la comunidad universitaria, tanto para su visualización como para la optimización de los aprendizajes (Naval y Ruiz Corbella, 2012). Este proceso se ha 
agudizado desde finales de los años noventa, una vez reestructuradas las universidades europeas, debido a creación del Espacio Europeo de Educación Superior.

Tiene también especial importancia en el ámbito español la promulgación de la Ley estatal del Voluntariado (Ley 6/1996, de 15 de enero, del Voluntariado) porque es a partir de ella que las universidades han comenzado a incorporar, en su oferta de extensión universitaria, servicios y/o programas que favorecen la participación del alumnado en proyectos de su entorno.

Como comentábamos, aún es reciente la participación del alumnado en diferentes espacios universitarios. Inicialmente esta participación se ha dado al margen de las actividades académicas, principalmente en actividades culturales o deportivas ligadas a la institución. Desde hace ya varios años, las universidades del Estado comenzaron a integrar dentro de sus estructuras, y de forma más o menos estable, servicios o programas que tienden a favorecer la participación de la comunidad universitaria dentro de las actividades y propuestas desarrolladas por las entidades de acción social existentes en los entornos más próximos de sus campus. Estos son los servicios de voluntariado, de extensión universitaria o de participación, por mencionar algunas de las denominaciones que han recibido (Arias y Simón, 2004).

Este proceso no ha sido azaroso. El propio contexto internacional parece haberse hecho más sensible a temas éticos en todos los niveles educativos, incluyendo el universitario; Kymlicka (2003) define este fenómeno como «ascenso del civismo». Para Bowen (1984) los departamentos, facultades o escuelas universitarias, teniendo como referencia la responsabilidad social, asumen dos grandes compromisos: uno técnico y otro moral. La responsabilidad técnica, en tanto que proporciona profesionales técnicamente competentes a la sociedad, bien entrenados, autodisciplinados y con un claro interés por seguir aumentando su aprendizaje a lo largo de su vida. Y, por otro lado, la responsabilidad moral, aludiendo a la formación de personas con conocimientos y cultura extensa, con capacidad para asumir liderazgo en la sociedad, amén de ejercer una influencia constructiva en la comunidad y en la vida cívica.

Por lo tanto, la educación superior debe preparar a sus alumnos de tal manera que lleguen a ser ciudadanos reflexivos, críticos y capaces de pensar autónomamente (Ugarte y Naval, 2010). En otras palabras, las instituciones educativas han de preparar para la participación e implicación social. Ya en los años setenta del siglo pasado, Otero $(1974,110)$ abogaba por un educando participante, considerando que la "toma decisiones implica problemas de motivación y actitud y problemas de conocimiento". El hecho de tener que discernir entre varias alternativas de procedimiento, y saber las consecuencias que puede traer su decisión, implica un mayor conocimiento de las variables; $y$, por consiguiente, la necesidad de acceder a la información. 


\section{APRENDER Y SERVIR DESDE LA UNIVERSIDAD. COMPETENCIA Y COMPROMISO}

Cualquier búsqueda de una correspondencia operativa para esa pedagogía del compromiso cívico en la educación superior conduce irremisiblemente al aprendizaje-servicio (nominación que preferimos por ser la más acuñada en la escena internacional), una estrategia o modalidad de intervención pedagógica ajustada, en filosofía y metodología, a los propósitos de una universidad implicada en la comunidad y abierta al mundo.

Como es perfectamente imaginable, su definición en la literatura temática se muestra tan dilatada que conviene fijar posición al respecto. Nos valemos de un trabajo de Bringle y Hatcher (1996, 222) porque responde suficientemente a esa expectativa: "Vemos el aprendizaje-servicio como una experiencia educativa donde los estudiantes participan en una actividad organizada de servicio que satisface necesidades comunitarias, haciéndolo de tal modo que consiguen ampliar su conocimiento de una materia, el aprecio de la disciplina y un sentido de responsabilidad cívica». Otra definición muy utilizada es la de Puig (2009, 9), quien define el ApS como «Una metodología que combina en una sola actividad el aprendizaje de contenidos, competencias y valores con la realización de tareas de servicio a la comunidad. En el aprendizaje-servicio el conocimiento se utiliza para mejorar algo de la comunidad y el servicio se convierte en una experiencia de aprendizaje que proporciona conocimientos y valores». En resumen, cabe decir que el aprendizajeservicio es una metodología experiencial que aúna aprendizaje y servicio comunitario en un único proyecto con base cívica y académica.

Ahora bien, no debe confundirse con el voluntariado ya que, a diferencia de este, el aprendizaje-servicio ha de formar parte del currículo, esperándose que produzca los mejores resultados cuando el servicio a realizar se relaciona con metas de aprendizaje (Puig, Batlle, Bosch y Palos, 2007). Permítasenos insistir en esto porque la apariencia de similitudes no confiere validez a los parangones. De modo más contundente, si cabe, el aprendizaje-servicio nada tiene que ver con el voluntariado desde el momento en que la generosidad personal dista de ser aquí la divisa nuclear de su motivación y activación; en tanto que el ApS requiere una sistematización de los aprendizajes en relación al servicio (Rodríguez Gallego, 2014).

Para una mejor aclaración terminológica y conceptual se presenta la siguiente figura. Como se puede ver, una vez fijados foco de atención y beneficiarios, se sitúan diferentes tipos de metodologías experienciales. 
FIGURA 1

Tipos DE METODOLOGÍAS EXPERIENCIALES

RECEPTORES

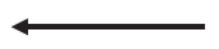

BENEFICIARIO

PROVEEDORES

SERVICIO

\section{FOCO DE ATENCIÓN}

APRENDIZAJE

APRENDIZAJE-SERVICIO

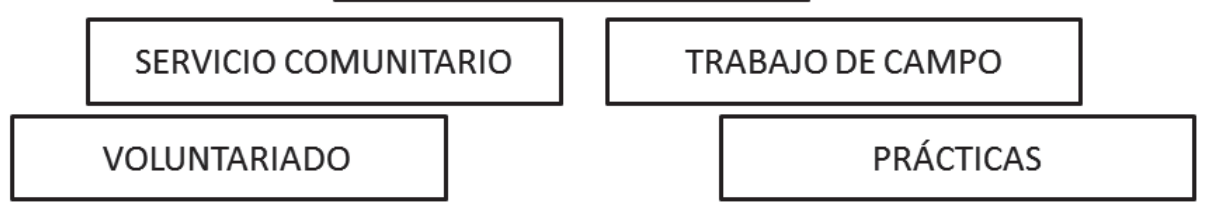

FuENTE: Adaptación de FuRCo (1996, 4).

Lo que ahora conviene es que nos preguntemos sobre el nivel de instalación del aprendizaje-servicio en el marco universitario, los elementos de su progresiva institucionalización y los factores que informan una dinámica que, en modo alguno, puede aventurarse sencilla. Haciéndonos eco de las reflexiones de Holland (2000) en torno al tema, no es suficiente con percatarnos de la potencialidad de la estrategia para el logro de cambios internos en centros y departamentos, sino que también precisamos conocer qué asuntos de la misma institución se relacionan con la exploración, implementación, expansión y sostenibilidad del aprendizaje-servicio en términos programáticos (Santos Rego, Sotelino y Lorenzo Moledo, 2015).

Esa tarea nos la ha facilitado un especialista en el tema a través de uno de sus últimos trabajos de revisión (Furco, 2007). Desde ahí resumimos la situación y extraemos algunas consecuencias. Su aportación descansa en un estudio exploratorio sobre la institucionalización del aprendizaje-servicio, utilizando para ello una muestra de la literatura existente y cuarenta y tres (43) universidades que han aplicado un mínimo de tres años este tipo de pedagogía en sus campus.

De lo que se trataba era de identificar factores críticos de institucionalización valiéndose para ello de procedimientos cuantitativos y cualitativos. De todos modos, la meta del análisis no era tanto llegar a conclusiones definitivas como averiguar patrones de diseño y acción que pudieran justificar posteriores estudios experimentales. Tres son los niveles de institucionalización acordados: a) construcción de masa crítica; b) calidad en el proceso; y c) institucionalización sostenida. A propósito del trabajo de Furco (2007), lo que nos interesa es trasladar los resultados en las cuatro áreas definidas para dar cuenta de la situación. En su inicio, el análisis gira en torno a la misión institucional y los propósitos del 
aprendizaje-servicio, destacándose la considerable heterogeneidad de metas declaradas por las universidades.

Las razones esgrimidas para institucionalizar la estrategia abarcan, entre otras, la mejora de la enseñanza en los estudios de grado, la construcción de comunidades de aprendizaje, la interdisciplinariedad, el fortalecimiento de las relaciones con la comunidad o el estímulo a la colaboración entre departamentos. La variedad propositiva repercute, lógicamente, sobre el enfoque con el que se administra la propia intervención pedagógica desde unidades más genéricas o más concretas, con mayor o menor gestión de los estudiantes en la planificación (Santos Rego, 2013).

Si atendemos a criterios de calidad o excelencia académica son varios los autores que apoyan el aprendizaje-servicio como un avance al respecto (Eyler y Giles, 1999; Herrero, 2002; Gallini y Moely, 2003; Hart y King, 2007; Bridgeland, Dilulio y Wulsin, 2008; Simonet, 2008; Conway, Amel y Gerwien, 2009; Leimer, Yue y Rogulkin, 2009; Tapia, 2008, 2012; Warren, 2012; Lockeman y Pelco, 2013). Su argumento básico está centrado en la posibilidad real de lograr impactos formativos de primer orden en los participantes. Valiéndonos de los enumerados por Tapia (2012), llamamos la atención sobre los siguientes:

- Impactos en el aprendizaje: mayor desarrollo de conocimientos conceptuales y competencias; destacando la puesta en práctica de la capacidad de análisis y síntesis de información compleja. El efecto sobre el aprendizaje no se produce de un modo directo, ya que diferentes trabajos publicados en este sentido apuntan a que un incremento del rendimiento tiene lugar por influencia de otras variables, caso de la autoestima o el empoderamiento (Strage, 2000; Gallini y Moely, 2003; Smink y Reimer, 2005; Lockeman y Pelco, 2013; Mella, Santos Rego y Malheiro, 2015).

- Impactos en el desarrollo de competencias para la inserción en el mundo laboral: Estas experiencias suponen una oportunidad para conectar con diferentes entidades y agentes en el ámbito laboral propio, lo que favorecerá, seguramente, una mejor consideración en su entorno profesional (Naval y Arbués, 2016). Además, estas experiencias contribuyen normalmente a desarrollar competencias básicas para trabajar en equipo, comunicarse eficazmente, asumir responsabilidades, desarrollar la iniciativa personal y aprender a organizar y gestionar recursos, dimensiones que llevan tiempo ganando terreno en acreditados planteamientos de inserción laboral (Tejada, 2013; Deeley, 2014; Glazier, Able y Charpentier, 2014; Coffey y Lavery, 2015).

- Impactos en la formación ética. Los proyectos de aprendizaje-servicio permiten la práctica de toma de decisiones profesionales, pero también éticas, para lo que sería ingenuo prescindir de las correspondientes pautas de reflexión y argumentación, de normas y costumbres en las diversas 
comunidades (Martínez Martín, 2008). En definitiva, permiten poner de manifiesto compromisos personales concretos (Furco, 2005).

- Impactos en la participación social y política. El aprendizaje-servicio reconoce la democracia como soporte cívico fundamental de participación, con consecuencias en el aprendizaje (Herrero, 2002). Asimismo, la profesora Vázquez Verdera (2015) indica que el aprendizaje-servicio puede ser un catalizador que facilite la formación de competencias para la acción transformadora que busca la construcción de realidades más sostenibles y transformadoras. Se refuerzan así los valores propiamente cívicos mediante el pensamiento crítico, el discurso público, las actividades grupales y, por supuesto, los vínculos de lo que se proyecta, y se hace, con la comunidad (Root, Callahan y Billig, 2005).

Tras la exposición de estos argumentos cabe señalar que el aprendizaje-servicio se postula como una vía, que puede ser académicamente eficaz, para poder ejercer la responsabilidad social de la universidad, contribuyendo de paso a formar ciudadanos honestos y solidarios. Ahora bien, su consecución no será posible solo con las buenas intenciones de pedagogos y profesionales en los distintos campos de conocimiento (Sotelino, 2015). Lo que realmente ayudará serán los operativos políticos y académicos de la universidad (Santos Rego, 2016).

En cuanto al segundo de los grandes apartados, mucho más centrado en las cuestiones académicas, no se puede decir que hubiera grandes sorpresas. El más crítico (por fundamental) de los elementos encontrados, muy por encima de los estudiantes y de los departamentos, tiene que ver con el profesorado y, más específicamente, con el conocimiento y las oportunidades que perciben en relación al aprendizaje-servicio dentro de su trayectoria académica. Sin olvidar una consistencia realmente notoria: la aceptación o el apoyo institucional y la aceptación o el apoyo de los académicos van de la mano.

Apoyaremos esta tesis sirviéndonos de un trabajo presentado en la Universidad de Santiago de Compostela (Sotelino, 2014), cuyo problema de investigación se trazaba en términos de viabilidad y posibilidad de implementación del aprendizaje-servicio en el ámbito universitario de Galicia. En respuesta al mismo, se optó por un estudio de corte cuantitativo. Siendo el primero de tal orientación en esta comunidad autónoma, se valoró la situación real de partida, para delimitar así el estado de la cuestión. Desde ahí se procedió a la evaluación usando una metodología ex-post-facto (no experimental), con un método descriptivo de encuesta entre el profesorado universitario.

La población no ha sido otra que el profesorado de la Universidad de Santiago de Compostela, en sus tres campus (dos en Santiago de Compostela y uno en la ciudad de Lugo). La muestra, constituida por 326 sujetos, se dividía en diferentes estratos de afiliación por área de conocimiento, quedando finalmente del siguiente modo: 60 profesores de Ciencias de la Salud, 52 de Ciencias Experimentales, 132 de Ciencias Sociales y Jurídicas, 33 de Enseñanzas técnicas y 47 de Arte y Humanidades. 
Como instrumento se optó por el más utilizado en la investigación por encuesta, esto es, un cuestionario con una batería de 45 ítems, estructurados en 5 bloques temáticos (perfil biográfico y profesional, práctica docente, compromiso social universitario, posibilidades metodológicas y cuestiones referidas al aprendizajeservicio). Cabe resaltar que el cuestionario incluía, a modo introductorio, una breve definición de aprendizaje-servicio. Dicho cuestionario ha alcanzado una fiabilidad que supera el 0,70 en todos los bloques, por lo que se considera aceptable según el índice alfa de Cronbach.

Dentro de este instrumento se preguntaba precisamente por el conocimiento y pertinencia del aprendizaje-servicio (ver Figura 2). Las respuestas a este cuestionario confirman que la mayoría los profesores desconocen tanto la metodología como alguna experiencia de ApS (68,1\% y 87,9\%, respectivamente). Aunque consideremos elocuente que el 31,3\% de ellos hayan escuchado hablar del aprendizajeservicio, tan solo un 12,1\% tiene conocimiento de alguna experiencia concreta.

Lo primero que se observa es que el profesorado valora positivamente $(89,8 \%)$ la incorporación de esta metodología a la universidad. Pero conviene destacar el apreciable número de sujetos que han dejado de responder al ítem (21,8\%). Dado su desconocimiento, abundan los que no se comprometen con respuesta alguna.

FIGURA 2

CONOCIMIENTO Y PERTINENCIA DEL APRENDIZAJE-SERVICIO

\begin{abstract}
¿Escuchó hablar con anterioridad de la metodología aprendizajeservicio (service-learning)?

¿Conoce alguna experiencia que relacione aprendizaje con servicio a la comunidad en el marco de...

¿Cree conveniente la incorporación de este tipo de metodología de ApS en la Universidad?
\end{abstract}

$$
\square \text { Sí } \quad \text { No }
$$

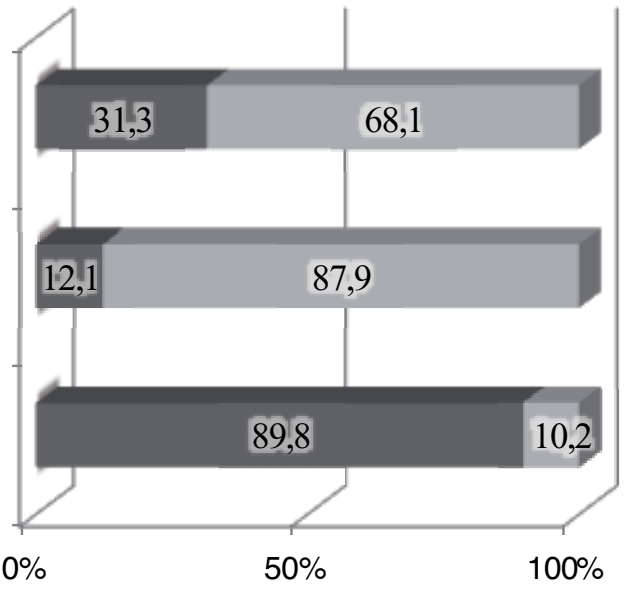

FuENTE: SOTELINO (2014).

Retomando el discurso de este apartado, pasaremos ahora a analizar la tercera área en el examen de los elementos potencialmente ligados a la institucionalización (participación y acuerdos con la comunidad). Son mayoría las recomendaciones 
que focalizan en los campus la dinámica de implementación del aprendizaje-servicio. No obstante, por más que la responsabilidad de contar con estructura y plan de actividad corresponda a la universidad, es obvio que sin la parte comunitaria dispuesta al efecto no hay sostenibilidad posible para el aprendizaje-servicio. Razón de más para no desatender en la investigación la forma en que este aspecto contribuye, o no, al asentamiento del aprendizaje-servicio en la educación superior.

La cuarta de las áreas contempladas aludía a las condiciones estructurales que pueden hacer sostenible el aprendizaje-servicio en el actual contexto universitario. En tal sentido, los campus que tienen creado un centro coordinador de las actividades muestran progresos y ganancias más significativas que los que aún carecen de tal estructura vertebradora; sirvan como ejemplo los casos de las universidades de Indianápolis, Stanford, Michigan o Minnesota en EE. UU. (Santos Rego, 2013); del Instituto Tecnológico de Monterrey en México (Benavides, 2011); de la National University of Ireland (Mcllrath, 2016); o, ya en nuestro país, de la Universidad de Barcelona y la Universidad Rovira i Virgili (Campo, 2014). En cuanto a su dependencia orgánica dentro de la institución, es más frecuente encontrar al aprendizaje-servicio bajo la responsabilidad y las competencias de la instancia encargada de regir los asuntos académicos.

Otro factor estructural que viene a reforzar el ya señalado rol del profesorado en la evolución del proceso es la aprobación, o no, dentro de la universidad de sistemas de apoyo y promoción, a fin de reconocer el ánimo y la voluntad de compromiso por parte del cuerpo docente (Rimmerman, 2009). Complementariamente, el estudio avala la conveniencia de establecer algún tipo de entidad, que asuma la responsabilidad de marcar estándares académicos y de servicio, dada la importancia de evitar distorsiones en el dispositivo pedagógico o abruptos descensos de calidad en el proceso.

En el trabajo anteriormente citado (Sotelino, 2014) también se interrogó al profesorado acerca de las principales trabas o problemas en la incorporación del aprendizaje-servicio a su docencia (ver Figura 3). Los más citados han sido los relacionados con la función docente: tiempo de los profesores $(60,5 \%)$, falta de formación (59\%) y motivación del profesorado (52,6\%); lo que nos sugiere que el profesorado parece asumir como responsabilidad propia el desarrollo de la metodología. Cabe destacar que el problema más señalado por los docentes de la muestra ha resultado la falta de apoyo económico a las iniciativas (62,6\%), aunque en muchos casos este tipo de experiencias apenas requieran de gasto alguno.

Algo insólito puede ser el dato de que la mayoría de los profesores no aprecie problemas atribuibles al alumnado a la hora de poner en marcha un proyecto de esta índole. Tan solo el 26,8\% se fija en el seguro de los estudiantes como un problema, el $25,2 \%$ en los permisos, el 36,6\% en la motivación y el $25,6 \%$ en la evaluación. Destacamos como excepción el tiempo de dedicación al proyecto, donde un $49,3 \%$ sí reconoce que podría haber un problema. Esto parece indicar que las experiencias que se están llevando a cabo en las universidades no producen reparos ni obstáculos por parte de los estudiantes a la implantación de este tipo de estrategias formativas. El atractivo de su diseño teórico alienta, en general, favorables expectativas (Sotelino, 2015). 
Han sido una minoría los que han marcado otras dificultades $(1,5 \%)$, destacando, en algunos casos, la excesiva burocracia o la falta de institucionalización. Precisamente, este dato guarda relación con el hecho de que sean pocos $(37,2 \%)$ los que aprecian como problema el bajo interés de las autoridades académicas hacia el Aps.

FIGURA 3

PROBLEMAS DE LA INCORPORACIÓN DEL APS

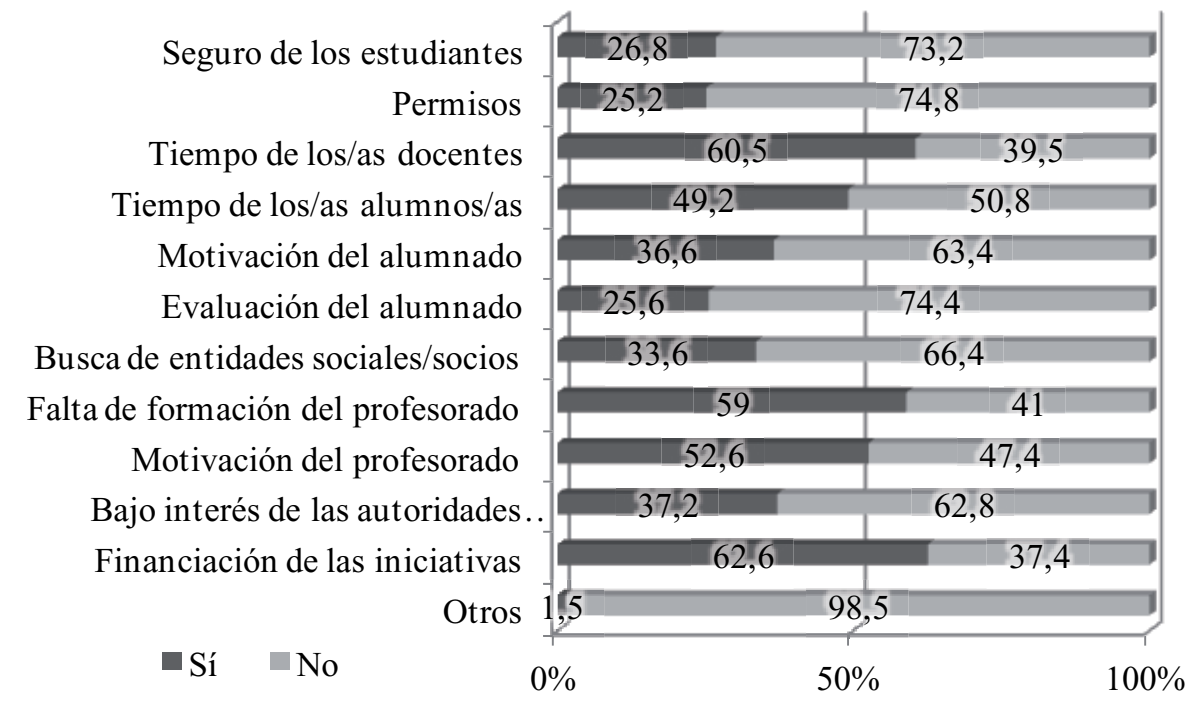

FUENTE: SOTELINO (2014).

Vistos en su conjunto, estos elementos críticos pueden resultar sugerentes al poner al descubierto parte del entramado institucional y social que es preciso conocer muy bien para que el aprendizaje-servicio pueda pensarse, diseñarse y activarse en una universidad. Y destapan un interrogante no menos pertinente acerca del tiempo necesario para culminar un proceso de esta naturaleza. La respuesta deviene nítida: son bastantes años de esfuerzo sostenido los que requiere una institucionalización plena, que podríamos estimar en una década, toda vez que no resulta fácil pasar de una fase o nivel al siguiente. Lo cual a nadie puede sorprender, por poco que sepa de la universidad y el ritmo de los cambios en su seno.

\section{CONCLUSIONES}

No deja de ser paradójico que en bastantes sistemas de educación superior, incluido el español, se muestre admiración (véanse, si no, los destinos de sus 
investigadores en formación y los 'séniors' en programas de movilidad) por el modelo de funcionamiento de las universidades norteamericanas y, sin embargo, les cueste reparar en que uno de los factores que han añadido calidad a su dimensionamiento académico es, precisamente, el de un 'compromiso cívico' entendido como soporte pedagógico extensivo de aprendizaje-servicio, al tiempo que medio con el que definir implicaciones en la sociedad civil y favorecer incluso la captación de fondos. Claro que la percepción de este y otros temas de interés cívico y moral se asocia a un cierto conocimiento previo y a una sensibilidad pedagógicosocial, que no siempre está repartida con equidad en el perfil de los académicos. Sea cual sea el país o la universidad en la que trabajen.

Pero no hemos de olvidar que los cambios aumentan sus probabilidades de éxito cuando se llevan a cabo según patrones de actividad culturalmente coherentes. Las culturas de los países y las culturas institucionales tienen peculiaridades que no se pueden despreciar pues, de lo contrario, estaríamos actuando bajo criterios de un mimetismo ingenuo, contra el que militamos, entre otras razones, porque no es receta que funcione.

Por otra parte, tengamos presente que la dimensión pedagógica de la acción universitaria se fortalece en la medida de su propulsión teórica y práctica a base de planteamientos clarificadores de la evolución que siguen los motivos y disposiciones cívicas de adolescentes y jóvenes (Ehrlich, 2000), o de cómo se hace recomendable una nueva estructuración del aprendizaje cuando se trata de integrar la noción de 'servicio' en el currículo ordinario de la titulación universitaria.

Es innegable que estamos inmersos en la gestión de un cambio derivado de la aún reciente implantación del EEEs, cuya prueba de fuego es conseguir que los alumnos se vayan haciendo dueños de su propio proceso de aprendizaje. Ello requiere de destrezas y actitudes que nutran de energía emocional su motivación e implicación en tareas y proyectos de carácter curricular y extracurricular.

Lo que importa es que los estudiantes lleguen a sentirse como agentes de ese cambio, e incluso puedan llegar a palpar sus efectos transformadores en una vecindad, en una escuela, en un hospital, en un taller, en una organización deportiva, en una comunidad de familias inmigrantes, o donde quiera que hayamos conseguido el despliegue de un programa de estas características. Es por ello que la universidad, como institución formadora, necesita de nuevas de técnicas, estrategias y metodologías pedagógicas que hagan partícipes a los educandos de tal responsabilidad, conectándolos con su entorno y fomentando el autoaprendizaje. De este modo, se propone el aprendizaje-servicio como una metodología válida y eficaz en la educación superior española. Así, los alumnos podrán aprender de necesidades que emanan de las propias realidades sociales, gestionando en primera persona la respuesta, algo que puede marcar su trayectoria vital y académica.

Sin embargo, hemos podido constatar que, mayoritariamente, el profesorado desconoce el aprendizaje-servicio y/o no encaja suficientemente experiencias realizadas en esa dirección. Ahora bien, el hecho de considerar en términos positivos la incorporación de esta metodología en las aulas sugiere que los cambios en la educación superior son posibles, siempre que se lleven a cabo sin maximalismos 
ingenuos y con modos inteligentes de incentivación del profesorado. Pensar y actuar para innovar, aprendiendo más y mejor. Esa podría ser la consigna o el lema para un tiempo de renovadas y renovadoras esperanzas en la universidad, como gran agencia de conocimiento y de ciudadanía.

De lo que se trata, en definitiva, es de encontrar soluciones pragmáticas que ayuden a consolidar el aprendizaje-servicio en las universidades españolas, adaptándolo a la idiosincrasia de nuestro modelo de educación superior. Lo cual ha de pasar por consensos para una cierta institucionalización de los programas y la previsión de dinámicas formativas adecuadas a cada contexto académico.

\section{REFERENCIAS BIBLIOGRÁFICAS}

ARIAS, S. y Simón, A. (2004) Las Estructuras Solidarias de las Universidades Españolas: Organización y Funcionamiento. Madrid, Universidad Autónoma de Madrid-Fundación Telefónica.

Barber, B. R. (1998) A passion for democracy. Princeton, Princeton University Press.

BENAVIDEs, E. (2011) Institucionalización de Políticas Educativas para una Educación Global. Revista Internacional sobre Investigación en Educación Global y para el Desarrollo, 0, 100-109.

Bowen, H. (1984) Graduate education and social responsibility. New Directions for Higher Education, 46, 113-119.

Bridgeland, J. M.; Dilulio, J. J. y Wulsin, S. C. (2008) Engaged for Success. Service-Learning as a Tool for High School Dropout Prevention. Washington, Civic Enterprises.

BRINGLe, R. G. y Hatcher, J. A. (1996) Implementing service learning in higher education. Journal of Higher Education, 67 (2), 221-239.

CABRERA, L. J. (2000) Educación y desarrollo. Revista de Educación, 322, 211-245.

CAMPO, L. (2008) El aprendizaje-servicio en la Universidad como propuesta pedagógica, en Martínez, M. (ed.) Aprendizaje-servicio y responsabilidad social de las universidades. Barcelona, Octaedro, 81-91.

CAMPO, L. (2014) Aprendizaje servicio y educación superior. Una rúbrica para evaluar la calidad de proyectos. Tesis doctoral inédita. Barcelona, Universitat de Barcelona.

COFFEY, A. y LAVERY, S. (2015) Service-learning: a valuable means of preparing pre-service teachers for a teaching practicum. Australian Journal of Teacher Education, 40 (7), 86-101.

COMISIÓN DE LAS COMUNIDAdes EuROPEAS (2006) Competencias clave para el aprendizaje permanente: Un Marco de Referencia Europeo. Luxemburgo, Oficina de Publicaciones Oficiales de las Comunidades Europeas.

Conway, J.; Amel, E. y Gerwein, D. P. (2009) Teaching and Learning in the social context: A Meta-Analysis of Service-Learning's Effects on Academic, Personal, Social and Citizenship Outcomes. Teaching of Psychology, 36, 233-245.

DeEley, S. J. (2014) Summative co-assessment: A deep learning approach to enhancing employability skills and attributes. Active Learning in Higher Education, 15 (1), 39-51.

Delors, J. (1994) Libro Blanco sobre Crecimiento, Competitividad y Empleo. Retos y pistas en el siglo XXI. Bruselas, Comisión Europea.

EHrLich, T. (2000) Civic responsibility and higher education. Phoenix, Az, Oryx Press.

EYLER, J. y GILES, J. (1999) Where's the learning in service-learning? California, Jossey-Bass Publishers. 
FURCO, A. (1996) Service-learning: a balanced approach to experiential education, en TAYLOR, B. y Corporation For National Service (eds.) Expanding Boundaries: Serving and Learning. Washington DC, Corporation for National Service, 2-6.

FurCO, A. (2003) Service-learning: A balanced approach to experiential education, en CAMPus COMPACT Introduction to Service-Learning Toolkit, 2 ed., 9-18. Recuperado de: http:// www.shsu.edu/academics/cce/documents/Service_Learning_Balanced_Approach_To_ Experimental_Education.pdf.

Furco, A. (2005) Impacto de los proyectos de aprendizaje-servicio, en Programa NACIONAL EDUCACIÓN SOLIDARIA, MECT Aprendizaje y servicio solidario en la Educación Superior y en los sistemas educativos latinoamericanos. Buenos Aires, Actas del séptimo Seminario Internacional Aprendizaje y Servicio Solidario, 19-26.

FurCO, A. (2007) Institutionalising service-learning in higher education, en MCILRATH, L. y LABHRAINN, I. M. (eds.) Higher education and civic engagement: international perspectives. Aldershot, Ashgate, 65-82.

GAETE, R. (2011) La responsabilidad social universitaria como desafío para la gestión estratégica de la Educación Superior: el caso de España. Revista de Educación, 355, 109-133.

Galuini, S. M. y Moely, B. (2003) Service-Learning and Engagement, Academic Challenge, and Retention. Michigan Journal of Community Service-Learning, 10 (1), 5-14.

GarCía Roca, J. y Mondaza CANAL, G. (2002) Jóvenes, universidad y compromiso social. Una experiencia de inserción comunitaria. Madrid, Narcea.

Glazier, J.; Able, H. y Charpentier, A. (2014) The impact os Service-Learning on Preservice Profesionals' Dispositions Towards Diversity. Journal of Higher Education Outreach and Engagement, 18, 4, 177-198.

HART, S. M. y KING, J. R. (2007) Service-Learning and literacy tutoring: Academic impact on pre-service teachers. Teaching and Teacher Education, 23, 323-338.

HeRrero, M. (2002) El problema del agua. Un desafio para incorporar nuevas herramientas pedagógicas al aula universitaria. Tesis doctoral. Universidad de Buenos Aires, Buenos Aires.

Holland, B. (2000) Institutional impacts and organizational issues related to service-learning. Michigan Journal of Community Service-Learning (número especial), Fall, 54-60.

Jover, G. y GonZÁlez Geraldo, J. L. (2013) Recreación del Espacio Europeo de Educación Superior en el horizonte de la sociedad de la sabiduría: hacia un nuevo escenario docente. Education in the knowledge society (EKS), 14 (3), 5-24.

KymlicKa, W. (2003) La política vernácula. Nacionalismo, multiculturalismo y ciudadanía. Barcelona, Paidós.

LARran, M. (coord.) (2014) Análisis del nivel de implantación de políticas de responsabilidad social en las universidades españolas. Colección experiencias y resultados, 03. Gran Canaria, Conferencia de Consejos Sociales y Fundación Carolina.

Leimer, C.; Yue, H. y Rogulkin, D. (2009) Does Service-Learning help students succeed? Assessing the Effects of Service-Learning at California State University-Fresno. Institutional Research, Assessment and Planning. Recuperado de www.calstate.edu/.

Lockeman, K. y Pelco, L. E. (2013) The Relationship between Service-Learning and Degree Completion. Michigan Journal of Community Service-Learning, 20 (1), 18-30.

LORENZO, M. del M. (2012) La función social de la universidad y la formación del profesorado. Edetania: Estudios y Propuestas Socio-educativas, 42, 25-38.

LUCAS, N. (2009) The influence of integrative and interdisciplinary learning on civic engagement, en JACOBY, B. (ed.) Civic engagement in higher education. Concepts and practices. San Francisco, Jossey-Bass, 99-116. 
Martínez Martín, M. (2006) Formación para la ciudadanía y educación superior. Revista Iberoamericana de Educación (versión electrónica), 42, 85-102.

Martínez Martín, M. (ed.) (2008) Aprendizaje servicio y responsabilidad social de las universidades. Barcelona, Octaedro.

MARTÍNEZ-ODRIA, A. (2010) Service-learning o aprendizaje-servicio: la apertura de la escuela a la comunidad local como propuesta de educación para la ciudadanía. Bordón. Revista de Pedagogía, 59 (4), 627-640.

MCIlrath, L. (2016) Democratising Knowledge through Civic Engagement. The Case of National University of Ireland, Galway, en SAnTos Rego, M. Á. (ed.) Sociedad del conocimiento. Aprendizaje e innovación en la universidad. Madrid, Biblioteca Nueva, 176-196.

Mella, I.; Santos Rego, M. Á. y Malheiro, X. M. (2015) Aprendizaje-Servicio y rendimiento académico del alumnado universitario. Revista de Estudios e Investigación en Psicología y Educación, 12. Recuperado de http://revistas.udc.es/index.php/reipe/article/ view/569.

NAval, C. y ARBuÉs, E. (2016) El aprendizaje-servicio y la transición desde la educación superior al mundo del trabajo, en SANTOS Rego, M. Á. (ed.) Sociedad del conocimiento. Aprendizaje e innovación en la universidad. Madrid, Biblioteca Nueva, 219-240.

NAval, C. y Ruiz Corbella, M. (2012) Aproximación a la responsabilidad social universitaria. La respuesta de la universidad a la sociedad. Bordón. Revista de Pedagogía, 64 (3), $103-115$.

NúÑEz CuBERo, L. (coord.) (2015) Cultura emprendedora y educación. Sevilla, Editorial Universidad de Sevilla.

Otero, O. F. (1974) La participación en los centros educativos. Pamplona, Eunsa.

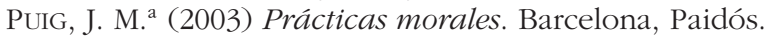

Puig, J. M. ${ }^{a}$ (coord.) (2009) Aprendizaje servicio (ApS). Educación y compromiso cívico. Barcelona, Graó.

Puig, J. M. ${ }^{a}$; Batlle, R.; Bosch, C. y Palos, J. (2007) Aprendizaje servicio. Educar para la ciudadanía. Barcelona, Octaedro.

Rimmerman, C. A. (ed.) (2009) Service-learning and the liberal arts. How and why it works. Lanham, Lexington Books.

Rodríguez Gallego, M. R. (2014) El Aprendizaje-Servicio como estrategia metodológica en la Universidad. Revista Complutense de Educación, 25 (1), 95-113.

Root, S.; Callahan, J. y Billig, S. H. (eds.) (2005) Improving Service-Learning practice. Research on Models to Enhance Impacts. Connecticut, Information Age Publishing.

SAIZ, J. E. y JimÉNEZ, S. R. (2008) Capital social: una revisión del concepto. Revista CIFE: Lecturas de Economía Social, 10 (13), 250-263.

SANJuRJO VIVO, V. (2012) El proceso de Bolonia: ¿mito y realidad? Revista Jurídica de Investigación e Innovación Educativa, 5, 121-146. Recuperado de http://www.eumed.net/ $\mathrm{rev} / \mathrm{rejie.}$

Santos Rego, M. Á. (ed.) (2010) Políticas educativas y compromiso social. El progreso de la equidad y la calidad. Barcelona, Octaedro.

Santos Rego, M. Á. (2013) ¿Para cuándo las universidades en la agenda de una democracia fuerte? Educación, aprendizaje y compromiso cívico en Norteamérica. Revista de Educación, 361, 565-590.

Santos Rego, M. Á. (ed.) (2016) Sociedad del conocimiento. Aprendizaje e innovación en la universidad. Madrid, Biblioteca Nueva. 
Santos Rego, M. Á. y Lorenzo Moledo, M. del M. (2007) Universidad y sociedad civil en Galicia. Vigo, Edicións Xerais.

Santos Rego, M. Á. y Lorenzo Moledo, M. del M. (2010) Dimensión cívica y desarrollo formativo de los estudiantes universitarios en el contexto español. Revista Electrónica de Investigación Educativa (número especial). Recuperado de http://redie.uabc.mx/ vol14no2/contenido-contenido.html.

Santos Rego, M. Á.; Sotelino, A. y Lorenzo Moledo, M. del M. (2015) Aprendizaje-servicio y misión cívica de la universidad. Una propuesta de desarrollo. Barcelona, Octaedro.

Simonet, D. (2008) Service-Learning and Academic Success: The Links to Retention Research. Western Michigan University. Recuperado de http://wmich.edu/servicelearning/ resources/articles.

SMINK, J. y REIMER, M. S. (2005) Fifteen effective strategies for improving student attendance and truancy prevention. Clemson: National Dropout Prevention Center. Recuperado de: http://www.dpi.state.nd.us/Title1/progress/present/15ways.pdf.

Sotelino, A. (2014) Aprendizaje-Servicio en las universidades gallegas. Evaluación y propuesta de desarrollo. Tesis doctoral inédita. Santiago de Compostela, Universidade de Santiago de Compostela.

Sotelino, A. (2015) Aprendizaje servicio y educación superior en Galicia. ¿Qué opinan los profesores? RIDAS, Revista Iberoamericana de Aprendizaje y Servicio, 1, 56-75.

SPRING, J. (2008) Research on globalization and education. Review of Educational Research, 78 (2), 330-363.

Strage, A. A. (2000) Service-Learning: Enhancing student learning outcomes in a collegelevel lecture course. Michigan Journal of Community Service Learning, 7, 5-13.

TAPIA, M. N. (2008) Calidad académica y responsabilidad social: el aprendizaje-servicio como puente entre dos culturas universitarias, en Martínez, M. (ed.) Aprendizaje servicio y responsabilidad social de las universidades. Barcelona, Octaedro, 27-56.

TAPIA, M. N. (2012) Academic Excellence and Community Engagement: Reflections on the Latin American Experience, en McIlrath, L.; LyOns, A. y MuncK, R. (eds.) Higher education and civic engagement. Comparative Perspectives. Nueva York, Palgrave Mcmillan, 187-203.

TEJADA, J. (2013) La formación de las competencias profesionales a través del aprendizajeservicio. Cultura y Educación, 25 (3), 285-294.

Ugarte, C. y Naval, C. (2008-09) El desarrollo de competencias cívicas en la universidad. Una experiencia de enseñanza online-presencial. Cuestiones Pedagógicas, 19, 115-140.

Ugarte, C. y Naval, C. (2010) Desarrollo de competencias profesionales en la educación superior. Un caso docente concreto. Revista Electrónica de Investigación Educativa, 12, 1-14. Recuperado de redie.uabc.mx/index.php/redie/article/view/251/412.

VÁzquez Verdera, V. (2015) El aprendizaje-servicio: una estrategia para la formación de competencias en sostenibilidad. Foro de Educación, 13 (19), 193-212.

VERNON, A. y Foster, L. (2002) Community agency perspectives in higher education. Service-learning and volunteerism en Service-learning through a multidisciplinary lens. Greenwich, IAP.

Warren, J. L. (2012) Does Service-Learning Increase Student Learning?: A meta-Analysis. Michigan Journal of Community Service-Learning, 18 (2), 56-61.

ZabalZA, M. Á. (2011) Metodología docente. REDU: Revista de Docencia Universitaria, 9 (3), 75-98. 